\title{
MTA2 promotes gastric cancer cells invasion and is transcriptionally regulated by Sp1
}

\author{
Chenfei Zhou ${ }^{1 \dagger}$, Jun $\mathrm{Ji}^{1+}, \mathrm{Qu} \mathrm{Cai}^{1}$, Min Shi ${ }^{1}$, Xuehua Chen ${ }^{1}$, Yingyan Yu', Bingya Liu', Zhenggang Zhu ${ }^{1}$ \\ and Jun Zhang ${ }^{1,2^{*}}$
}

\begin{abstract}
Background: MTA2 gene belongs to metastasis associated family, and is highly expressed in some solid tumors, including gastric cancer. Its biological function in gastric cancer is currently undefined.

Methods: Metastasis-associated tumor gene family 2 (MTA2) and transcription factor specificity protein 1 (Sp1) expression were detected in 127 gastric cancer samples by immunohistochemistry staining. SGC-7901 and AGS gastric cancer cell lines transfected by MTA2 shRNA was used for biological function investigation. Binding and regulation activities of Sp1 on MTA2 promoter were investigated by chromatin immunoprecipitation and luciferase reporter gene.

Results: The expression rate of MTA2 in gastric cancer tissues was 55.9\% (71/127), and its expression was closely related to the depth of tumor invasion, lymph nodes metastasis, and TNM staging. MTA2 knockdown in human SGC-7901 and AGS gastric cancer cells significantly inhibited migration and invasion in vitro, and disrupted structure of cytoskeleton. MTA2 knockdown also attenuated xenografts growth and lung metastasis in nude mice model. MTA2 expression was positively correlated with transcription factor Sp1 in gastric cancer tissues $(r=0.326, P<0.001)$. Sp1 bound to human MTA2 gene promoter at region from -1043 bp to -843 bp. Transcriptional activity of MTA2 promoter could be enhanced by Sp1 overexpression.
\end{abstract}

Conclusions: MTA2 knockdown impairs invasion and metastasis of gastric cancer cells, and attenuates xenografts growth in vivo. Sp1 regulates MTA2 expression at transcriptional level.

Keywords: Gastric cancer, MTA2, Cell invasion, Transcriptional regulation, Sp1

\section{Background}

Gastric cancer is one of the major malignancies in mainland of China, with high incidence and mortality [1]. The newly diagnosed gastric cancer patients in China account for $40 \%$ worldwide cases every year [2,3]. Furthermore, over $80 \%$ gastric cancer patients in this developing country are diagnosed at advanced stage, resulting in a relative poor outcome $[4,5]$.

Distant metastasis is the major cause of death for cancer patients. Initiation of metastasis consists of multiple steps

\footnotetext{
* Correspondence: jun_zj10977@163.com

${ }^{\dagger}$ Equal contributors

'Department of Surgery, Shanghai Institute of Digestive Surgery, Ruijin Hospital, Shanghai Jiao Tong University School of Medicine, No. 197 Ruijin er Road, Shanghai 200025, P.R. China

2Department of Clinical Oncology, Ruijin Hospital, Shanghai Jiao Tong University School of Medicine, No. 197 Ruijin er Road, Shanghai 200025, P.R. China
}

\section{Biomed Central}

(c) 2013 Zhou et al.; licensee BioMed Central Ltd. This is an Open Access article distributed under the terms of the Creative Commons Attribution License (http://creativecommons.org/licenses/by/2.0), which permits unrestricted use, distribution, and reproduction in any medium, provided the original work is properly cited. The Creative Commons Public Domain Dedication waiver (http://creativecommons.org/publicdomain/zero/1.0/) applies to the data made available in this article, unless otherwise stated. and involves abundant molecules events [6,7]. Mechanisms of metastasis in gastric cancer are still under investigation. Metastasis-associated tumor gene family (MTA) has three members, MTA1, MTA2 and MTA3. MTA1 overexpression was observed in some solid tumors, including breast, esophageal, pancreatic, hepatocellular carcinoma [8], and correlated with cancer cell invasion and metastasis $[9,10]$. MTA2 has high homology with MTA1 in protein alignment, and also consists in nucleosome remodeling and histone deacetylase (NuDR) complex $[11,12]$. These results suggested that function of MTA2 might be similar to MTA1. Currently, its biological function in gastric cancer is still unclear.

The regulation of MTA family has not been fully elucidated. It was reported that mouse Mta2 gene was regulated by transcription factor specificity protein 1 (Sp1) [13]. Although amino acid sequences of MTA2 between 
human and mouse are highly identical [11], the promoter sequences are different. Sp1 was found to be overexpressed in gastric cancer tissues, and had positive correlation with MTA2 [14-16]. Those results hinted potential regulatory role of Sp1 in MTA2 transcription.

In present study, the biological function of MTA2 protein in gastric cancer was assessed both in vitro and in vivo, the role of $\mathrm{Sp} 1$ in transcriptional regulation of human MTA2 gene promoter was also investigated.

\section{Results}

MTA2 protein was highly expressed in gastric cancer tissues The results of immunohistochemistry staining revealed that, the positive rate of MTA2 protein in gastric cancer tissues was $55.9 \%$ (71/127). MTA2 protein was mainly localized in the nuclear of gastric cancer cells, and its expression was much more dominant in tumor tissues than that in neighboring gastric mucosa (Figure 1A). MTA2 protein expression was closely related with depth of tumor invasion $(P=0.003)$, lymph nodes metastasis $(P=0.019)$, and TNM staging $(P=0.007)$, whereas no correlation with Lauren's classification and tumor differentiation (Table 1). Patients with MTA2 expression had a shorter overall survival time with marginally significance $(60 \mathrm{mos}$ vs. $76 \mathrm{mos}$, $P=0.078$, Figure $1 B$ ). By multivariate analysis, TNM staging was an independent prognostic factor $(P<0.001$, HR 2.165 , 95\% CI 1.612 to 2.909 ). However, no survival difference was observed in patients with different MTA2 status in identical TNM staging (Additional file 1: Figure S1).

\section{MTA2 knockdown attenuated migration and invasion of gastric cancer cells}

MTA2 expression in gastric cancer cell lines SGC-7901 and AGS was knocked down by shRNA transfection, and cells transfected by vector was identified as negative control (Figure 2A). In proliferation assay, no significant difference was observed between MTA2 knockdown group and control group. In colony formation assay, the number of clones was similar between two groups, but the size of clones formed in MTA2 knockdown cells was smaller than those in control group (Figure 2B).

The wound healing course of MTA2 knockdown group was significantly slower than that in control group. Furthermore, at the edge of scratch, pseudopodia of control cells could extend perpendicular to the free space of scratch, which was found in MTA2 knockdown group (Figure 2C). For SGC-7901 cells, the numbers of cells penetrating the membrane of chambers in both migration and invasion assays were significantly less in MTA2 knockdown group than those in control group (Migration: $3.0 \pm 1.7$ vs. $17.3 \pm 4.5, P=0.007$; Invasion: $3.7 \pm 1.5$ vs. $54.0 \pm 14.4, P=0.004$, Figure $2 \mathrm{D}$ ), respectively. For AGS cell line, the result of migration assay was similar ( $2.7 \pm 1.5$ vs. $13.7 \pm 2.1, P=0.002$, Figure $2 \mathrm{D})$, and in invasion assay, number of cells were low in both groups. MTA2 expression in AGS/shMTA2 cells was rescued by transient transfection of MTA2 plasmid. Migration ability of AGS/shMTA2 cells was partially recovered (Additional file 1: Figure S2).

\section{MTA2 knockdown disturbed the structure of actin cytoskeleton}

In order to confirm the morphological changes observed in wound healing assay, the structure of actin cytoskeleton was observed. In MTA2 knockdown cells, the structure of fibrous actin (F-actin) was less than cells in control group. Cell morphology was changed from spindle to spherical after MTA2 knockdown (Figure 3A). The expression of CD24 and MYLK, genes related with cytoskeleton regulation were assessed by qRT-PCR. These two genes were found to be significantly reduced in MTA2 knockdown cells than those in control group (Figure 3B).
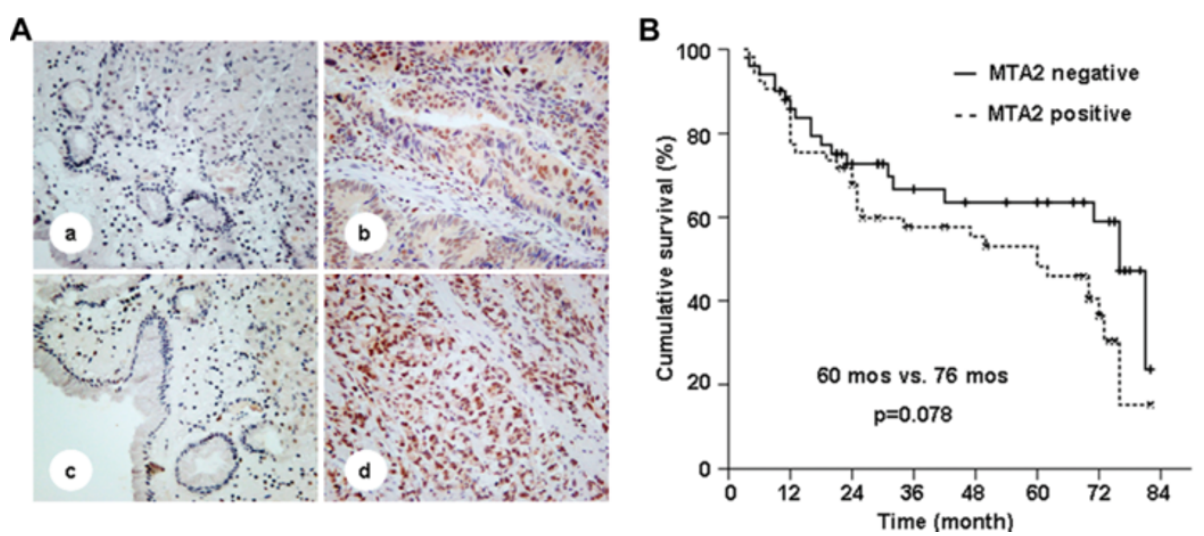

Figure 1 MTA2 in paired mucosa beside tumor and tumor tissues, and survival curve of patients. A, MTA2 expression in paired gastric mucosa beside tumor and tumor tissues from two patients ( $a, b$ and $c$, d) were detected by $1 \mathrm{HC}(\times 100)$. B, Patients with MTA2 positive expression had a shorter median overall survival than those with MTA2 negative ( 60 mos vs. 76 mos, $p=0.078$ ). 
Table 1 Correlation between MTA2 and clinicopathological characteristics of gastric cancer patients

\begin{tabular}{|c|c|c|c|}
\hline \multirow{2}{*}{$\begin{array}{l}\text { Clinicopathological } \\
\text { characteristics }\end{array}$} & \multicolumn{2}{|c|}{ MTA2 } & \multirow[t]{2}{*}{$P$} \\
\hline & - & + & \\
\hline \multicolumn{4}{|l|}{ Tumor invasion } \\
\hline $\mathrm{T} 1$ & 10 & 3 & \multirow{4}{*}{0.003} \\
\hline $\mathrm{T} 2$ & 15 & 8 & \\
\hline $\mathrm{T} 3$ & 25 & 50 & \\
\hline $\mathrm{T} 4$ & 6 & 10 & \\
\hline \multicolumn{4}{|l|}{ Lymph node metastasis } \\
\hline Negative & 28 & 21 & \multirow{2}{*}{0.019} \\
\hline Positive & 28 & 50 & \\
\hline \multicolumn{4}{|l|}{ TNM staging } \\
\hline 1 & 20 & 9 & \multirow{4}{*}{0.007} \\
\hline$\|$ & 13 & 14 & \\
\hline III & 13 & 32 & \\
\hline IV & 10 & 16 & \\
\hline \multicolumn{4}{|l|}{ Lauren's type } \\
\hline Intestinal & 28 & 31 & \multirow{2}{*}{$>0.05$} \\
\hline Diffuse & 28 & 40 & \\
\hline \multicolumn{4}{|l|}{ Differentiation } \\
\hline High & 16 & 15 & \multirow{2}{*}{$>0.05$} \\
\hline Low & 40 & 56 & \\
\hline
\end{tabular}

MTA2 knockdown attenuated xenograft growth and lung metastasis

The growth rate of MTA2 knockdown SGC-7901 xenografts was slower than that in control group $(P=0.043$, Figure $4 \mathrm{~A}$ ), and the average tumor weight of xenografts was also lower $(0.82 \pm 0.08 \mathrm{~g}$ vs. $2.12 \pm 0.58 \mathrm{~g}, P=0.021)$. MTA2 expression in xenografts was detected both by western blot (Additional file 1: Figure S3) and IHC (Figure 4B). Ki-67 expression was significantly reduced in MTA2 knockdown group, while apoptosis index $(3.3 \pm 0.6 \%$ vs. $3.0 \pm$ $1.0 \%, P=0.643)$ and microvessel density were similar in both groups (Figure 4B). In lung metastasis models, four mice in control group (4/9) developed pulmonary metastase (Figure 4C). Per contra, no mice were found to develop lung metastasis in MTA2 knockdown group (0/9).

\section{MTA2 in gastric cancer tissues was related with Sp1 expression}

Both MTA2 and Sp1 protein had similar expression pattern in gastric cancer tissues (Figure $5 \mathrm{~A}$ ). The expression rate of MTA2 was significant higher in those concomitantly with $\mathrm{Sp} 1$ expression (Table $2, P<0.01$ ). Positive correlation was found between the expression of these two proteins $(r=0.326, P<0.001)$. Concomitant expression of
MTA2 and Sp1 was also observed in gastric cancer cell lines (Additional file 1: Figure S4).

\section{Binding area of Sp1 on MTA2 promoter located at region from -1043 bp to $-843 b p$}

The results of ChIP assay in SGC-7901 cells demonstrated that, PCR product could be amplified by primer1 (-1179 bp to $-843 \mathrm{bp}$ ) and primer1.2 (-1043 bp to $-843 \mathrm{bp})$, rather than other 3 pairs. To confirm the in vitro results, ChIP assay was also performed using gastric cancer tissues with Sp1 high expression. PCR product could also be amplified by primer1.2 rather than primer1.1 (Figure $5 \mathrm{~B}$ ).

\section{Sp1 overexpression enhanced the transcriptional activity of MTA2 promoter}

Reporter plasmids were co-transfected into SGC-7901 cells with either Sp1 plasmid or vector. The activities of pGL3-1179, pGL3-1042, pGL3-797 and pGL3-482 were significantly elevated by $\mathrm{Sp} 1$ introduction, whereas the activity of pGL3-117 was extremely low, and had no response to Sp1 introduction (Figure 5C). Similar results were also demonstrated in AGS cells (Figure 5C). MTA2 mRNA expression was increased after $\mathrm{Sp} 1$ overexpression in MKN45 cells, whereas the protein level was not significantly changed (Additional file 1: Figure S5).

To verify the transcriptional role of Sp1 in MTA2 promoter, Sp1 specific siRNA was co-transfected into SGC7901 cells with pGL3-264, the reporter plasmid containing Sp1 binding area. Luciferase activity was significantly reduced by siRNA transfection after $24 \mathrm{~h}$ (Figure 5D).

\section{Discussion}

MTA2 is an essential part for NuRD complex and activity [17]. The pathological and biological characteristics of MTA2 in malignancies were rarely reported. MTA2 overexpression was first described in cervical cancer [11]. Hereafter, its aberrant expression was found in ovarian epithelial cancer, hepatocellular cancer, nonsmall cell lung cancer, and correlated with invasive phenotypes such as large tumor size, poor differentiation and lymph node metastasis [18-20].

The results of this study validated the patterns of MTA2 expression in gastric cancer tissues as we previously reported [16]. Whether MTA2 could be served as a prognostic factor of gastric cancer was still unclear [20]. Patients with MTA2 expression had a trend of worse outcome. However, survival analysis stratified by TNM staging showed no difference between MTA2 positive or negative groups (Additional file 1: Table S1). Therefore, even MTA2 expression was found to be related with TNM staging, it is still couldn't be considered an independent prognostic factor. 


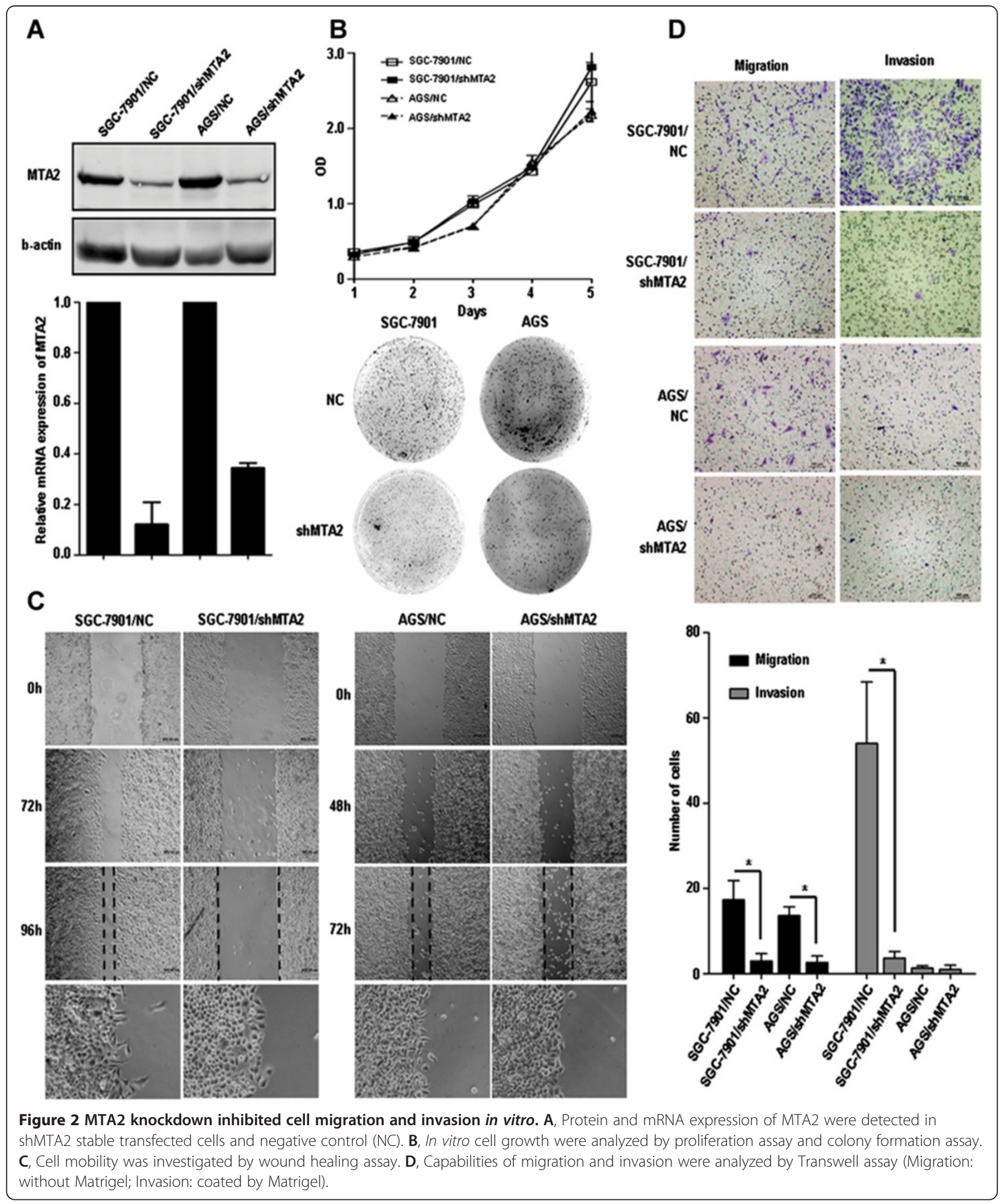

The process of cancer invasion and metastasis is complicated, tumor cells break through the barrier of normal tissue, invade into surrounding extracellular matrix and circulation, then extravasate into distant organs to form metastasis foci. Cell mobility and invasion ability play important roles in these processes [7]. The results of present study revealed the role of MTA2 in regulating gastric cancer cell mobility and invasion. 


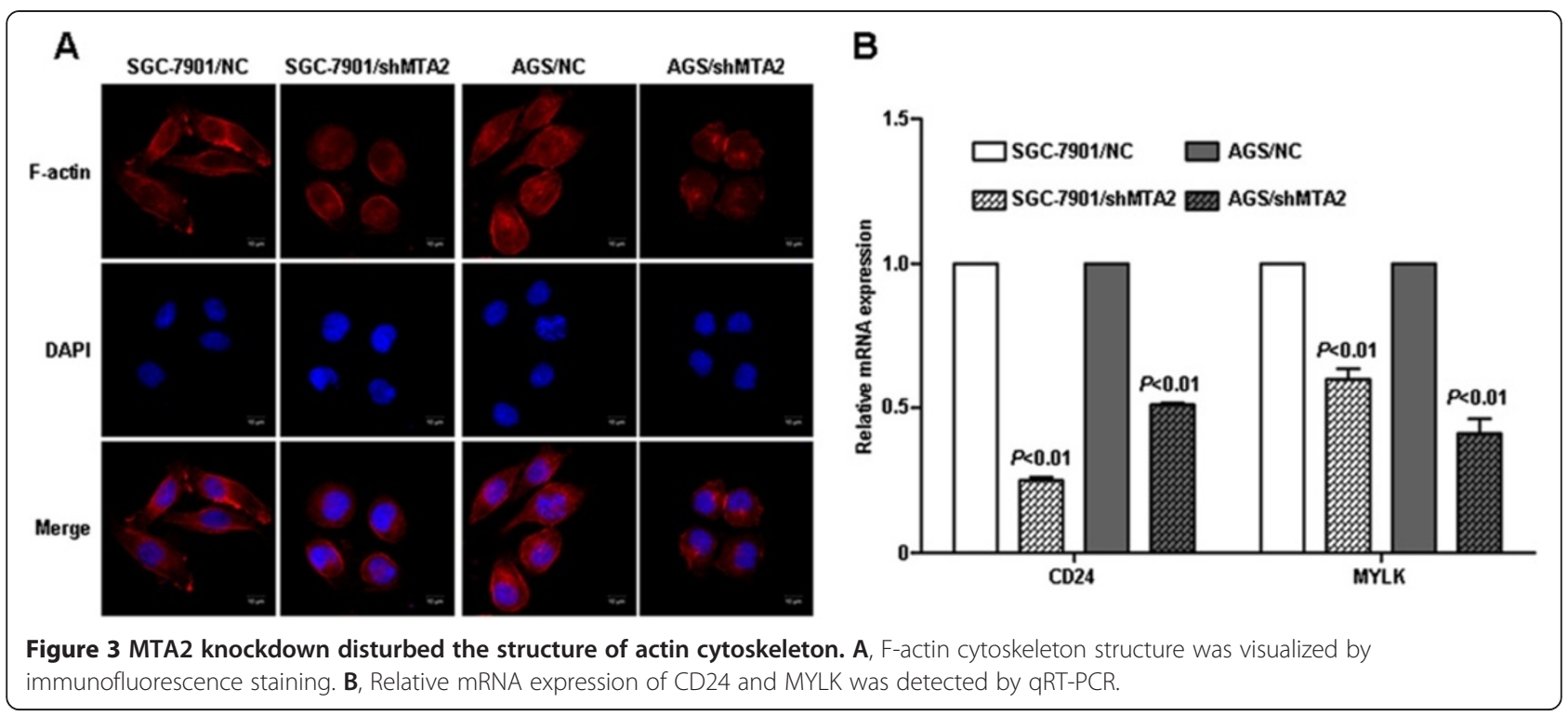

$\mathrm{Fu}$ et al. also found that invasion and metastasis abilities of mouse 4 T1 mammary tumor cells were reduced after Mta2 gene interference [21]. These results indicated that MTA2 might participate in the process of tumor progression.

The mechanisms of MTA2 in regulating cancer cell mobility and invasion were unclear. MTA2 was reported to serve as a component of Twist complex, and participated in inhibition of E-cadherin gene transcription. In this study, we found that knockdown of MTA2 gene expression could alter gastric cancer cell morphology and disturb F-actin structure. Furthermore, reduced mRNA expression of both CD24 and MYLK were found in MTA2 knockdown cells. Cytoskeleton remolding is essential for controlling cell motility and cancer invasion [22]. Up-regulation of CD24 could increase actin cytoskeletal remolding dynamic and enhance contractile forces to facilitate cancer cell invasion [23,24]. Myosin

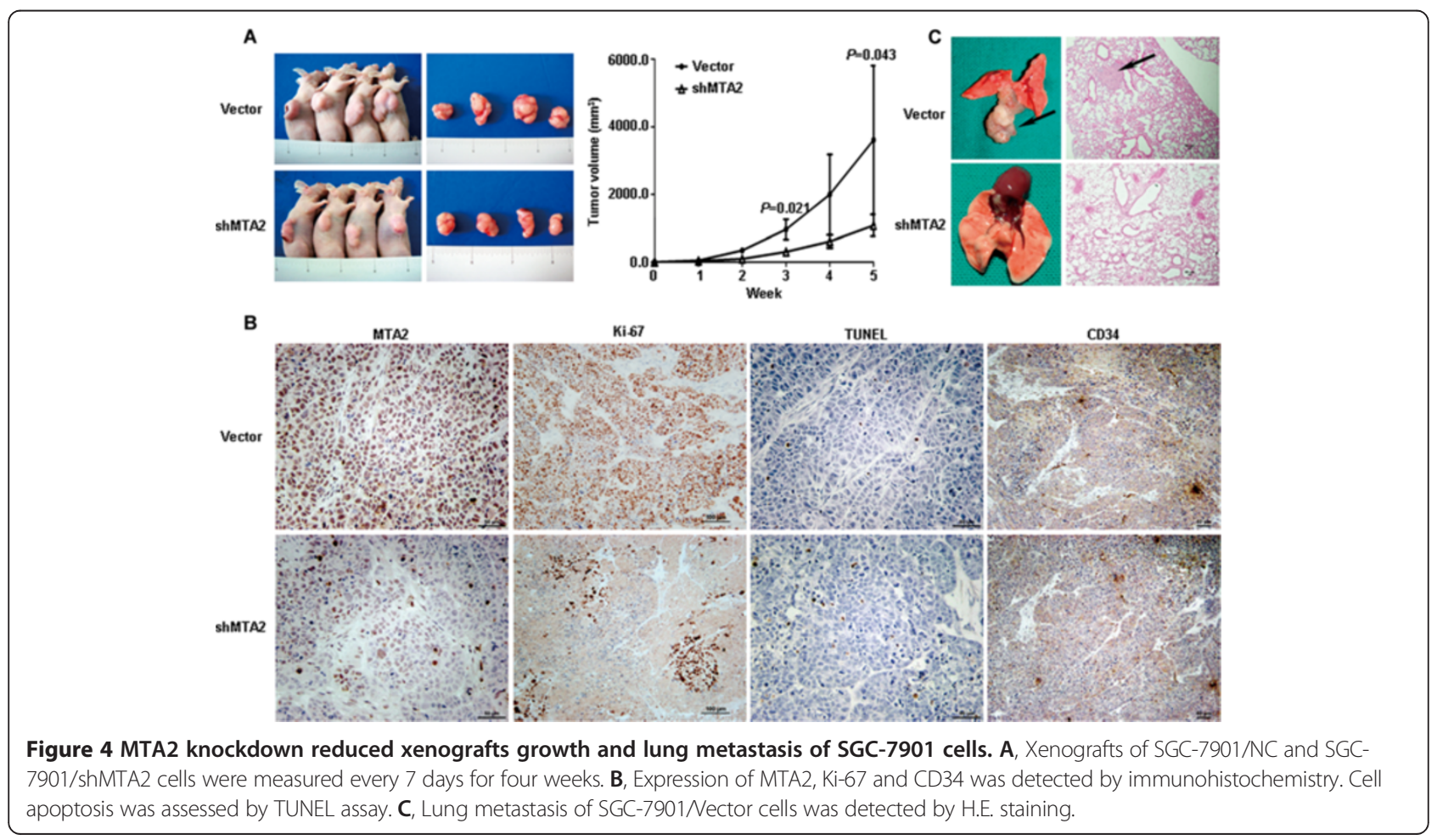




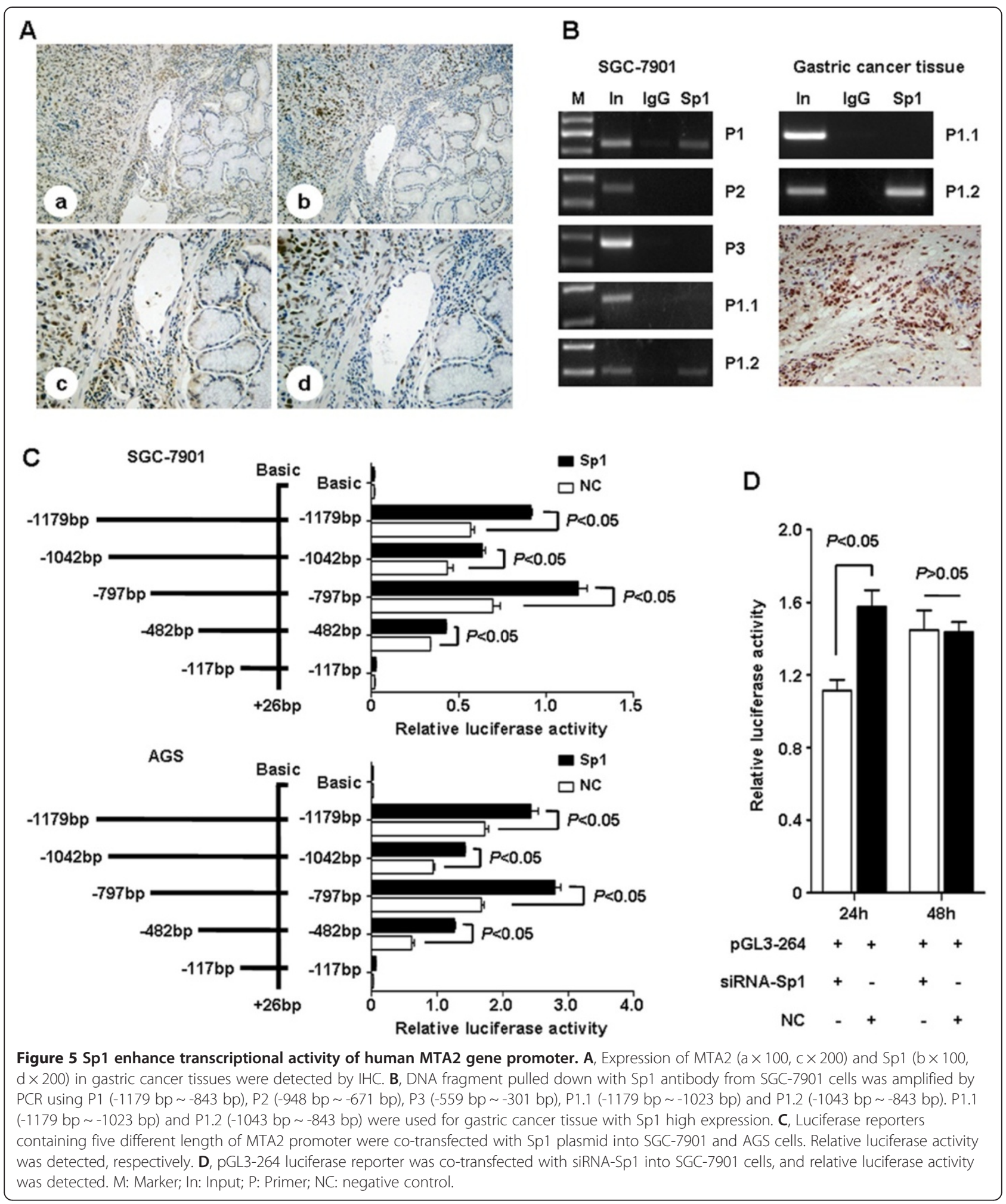

light chains phosphorylated by MYLK increase its interaction with actin filaments, and promote cell protrusion and contraction [25]. NuRD complex, which MTA2 consists in, regulates gene expression by remolding the structure of nucleosome. Alteration of CD24 and MYLK expression might be one of the mechanisms for MTA2 to modulate cytoskeleton and participate in cell invasion. 
Table 2 Expression of Sp1 and MTA2 in gastric cancer tissues

\begin{tabular}{|c|c|c|c|c|}
\hline & & \multicolumn{2}{|c|}{ MTA2 } & \multirow[t]{2}{*}{$P$} \\
\hline & & - & + & \\
\hline \multirow[t]{2}{*}{ Sp1 } & - & 35 & 20 & $<0.01$ \\
\hline & + & 21 & 51 & \\
\hline
\end{tabular}

The impacts of MTA2 knockdown on gastric cancer cell growth in vitro seemed to be a paradox. MTA2 knockdown didn't interfere gastric cancer cell growth in proliferation assay, but colony formation was attenuated. This divergence might be attributed to different culture conditions. Cells adhered to culture plates in proliferation assay, whereas was suspended in agar during colony formation. Adhesion to substratum is necessary for normal adhering cells survival and growth [26]. Tumor cells, however, can survive and grow in adhesion absent or anchorage-independent condition, which is important for tumorigenesis as well as metastasis [27]. Colony formation assay is a convenient assay to assess the capability of cell growth in anchorage-independent condition, and is closely related to the in vivo situation [28]. Attenuation of tumorigenesis and metastasis in vivo validated the result of colony formation assay, indicated that MTA2 knockdown could impair the anchorage-independent growth of gastric cancer cells. Exogenous expression of MTA2 in SaoS-2 cells could enhance cell growth in colony formation assay. Reducing of p53 acetylation and expression after MTA2 overexpression suggested that p53 inhibition might be one of the mechanisms of MTA2 regulating anchorage- independent growth [29]. Increased p53 expression was also found in MTA2 knockdown cells in this study (Additional file 1: Figure S6).

Transcription factor Sp1 plays an important role in physiological process and also in human cancer progression by regulating transcription of diverse downstream genes [30,31]. Concomitant expression of MTA2 and Sp1, and high GC-content sequence in proximal region of human MTA2 promoter, the potential Sp1 binding region [32], indicated that MTA2 might also be a downstream gene of Sp1 in gastric cancer. The results of ChIP and luciferase activity assays demonstrated that Sp1 could bind to MTA2 promoter, and enhance its transcriptional activity.

In ChIP assay, we found that the binding area of Sp1 on MTA2 promoter was at -1043 bp to -843 bp. However, promoter downstream of this fragment could also be regulated by Sp1 introduction. The characteristics of Sp1 might attribute to this phenomenon. As a critical transcription factor, Sp1 can regulate the expression of abundant genes, including many transcription factors, and even itself [33]. Overexpression of Sp1 might trigger the activities of some transcription factors, and increased the transcriptional activity of MTA2 promoter without direct binding.

On the other hand, multiple transcription factors typically bind together and form a transcriptional complex [34]. Alteration of one factor might not abrogate the function of the complex. Luciferase activity recovered at $48 \mathrm{~h}$ after Sp1 knockdown suggested a compensation mechanism might exist. Xia et al. reported that cooperation of transcription factor ETS or existence of Sp3 might contribute to stable Mta2 protein level in mouse ES cells after Sp1 knockdown [13]. In our study, several transcription factors' binding sites were predicted in fragment from -1042 bp to $-797 \mathrm{bp}$, including AP-1, NF-1 and E2F which were reported had interaction with Sp1 [30]. Although the regulatory function of $\mathrm{Sp} 1$ on MTA2 promoter was demonstrated in this study, the in-detailed mechanisms of MTA2 transcription need to be further clarified.

In conclusion, aberrant expression of MTA2 in gastric cancer cells participates in cell mobility and invasion. Overexpression of Sp1 could regulate the transcriptional activity of MTA2 promoter and might partially contribute to MTA2 expression in gastric cancer.

\section{Materials and methods}

\section{Ethical statement}

Written informed consent was obtained from all participants, and study protocol was approved by the ethics committee of Ruijin Hospital, Shanghai Jiaotong University School of Medicine. All mouse experiments were approved (Permit\#20110930) by the Animal Care and Use Committee and conducted an accord with the Guide for the Care and Use Laboratory Animals of Ruijin Hospital, Shanghai Jiaotong University School of Medicine.

\section{Patients and samples}

Paraffin-embedded specimens from 127 gastric cancer patients underwent standard D2 resection, were collected from Department of Surgery, Shanghai Ruijin Hospital. All samples were pathologically confirmed as gastric adenocarcinoma. Clinicopathological data were reviewed, and were listed in Additional file 1: Table S1. TNM staging classification was based on criteria of American Joint Committee on Cancer (AJCC, 6th edition). Overall survival was recorded from 103 patients.

\section{Cell lines and transfection}

Gastric cancer cell lines SGC-7901, AGS, MKN45, BGC823 were purchased from Shanghai Institute of Biological Sciences, Chinese Academy of Sciences. Hs746T was purchased from ATCC. Cells were cultured by RPMI-1640 medium with $10 \%$ fetal calf serum at $37^{\circ} \mathrm{C}$ and $5 \% \mathrm{CO}_{2}$. Five shRNA plasmids targeting different regions of MTA2 mRNA were purchased from Sigma. Plasmid shRNA-74 had the strongest efficiency, and its interference sequence 
was 5' -CCC TCT TGA ATG AGA CAG ATA CTC GAG TAT CTG TCT CAT TCA AGA GGG-3'. Stable transfected cells were selected by puromycin $(5 \mu \mathrm{g} / \mathrm{ml})$. Luciferase reporter plasmids constructed by pGL3-Basic vector (Promega) and truncated MTA2 promoters were co-transfected with Sp1 plasmid (persevered in our institute) into SGC-7901 and AGS cells. Relative luciferase activity was detected at $48 \mathrm{~h}$ after transfection by dualluciferase assay system (Promega). Ratio of reporter plasmids and control plasmid (pRL-TK, Promega) was 20:1. The concentration of Sp1-siRNA (Invitrogen) transfected into SGC-7901 cells was 50 nM. Lipofectamine 2000 (Invitrogen) was used as transfection regent.

\section{Immunohistochemistry staining (IHC)}

Immunohistochemistry staining was performed on $4 \mu \mathrm{m}$-thick slices following EnVision two-step procedure of Dako REAL ${ }^{\mathrm{Tm}}$ Envision $^{\mathrm{Tm}}$ Detection System (Dako). Slides were incubated by primary antibodies including MTA2 (1:200, Santa Cruz), Sp1 (1:200, Santa Cruz), Ki67 (1:50, Dako), and CD34 (1:150, Dako), respectively, followed incubated by HRP labeled secondary antibody and were visualized by diaminobenzidine.

Both MTA2 and Sp1 proteins were localized in cell nuclear stained as brownish granules. The expression status of MTA2 and Sp1 was determined by product score of average percentage and intensity of positive cells under 5 random high-power fields [14]. Score for percentage: $<5 \%(0)$, 5\%-25\% (1), 25\%-50\% (2), 50\%-75\% (3), and >75\% (4); for intensity: no staining (0), light brown (1), brown (2), and dark brown (3). For MTA2, score of 0 and $\geq 1$ was defined as negative and positive, respectively. For Sp1, score of $\leq 3$ and $>3$ was defined as negative and positive, respectively.

\section{Western blot}

One hundred micrograms protein were fractionated by 10\% SDS-PAGE gel and transferred to PVDF membranes. PVDF membranes were blocked by $5 \%$ skim milk for $1 \mathrm{~h}$, then were incubated overnight at $4^{\circ} \mathrm{C}$ with primary antibodies, including MTA2 (1:1000, BETHYL), Sp1 (1:500, Santa cruz) and HDAC1 (1:1000, Cell signaling technology). Fluorescent secondary antibodies (1:15000, LI-COR) and infrared imaging system (LI-COR) were used to visualize the protein bands.

\section{Quantitative reverse transcription-polymerase chain reaction (qRT-PCR)}

RNA was extracted by Trizol reagent method. Reverse transcription in $20 \mu$ l-system was preformed following protocol of Applied Biosystems. Primers for qRT-PCR were MTA2: F-TGT ACC GGG TGG GAG ATT AC, R-TGA GGC TAC TAG AAA TGT CCC TG; CD24: F-CTC CTA CCC ACG CAG ATT TAT TC, R-AGA GTG AGA CCA CGA AGA GAC; MYLK: F- CCC GAG GTT GTC TGG TTC
AAA, R-GCA GGT GTA CTT GGC ATC GT. Quantitative mRNA expression was measured by ABI Prism 7900HT sequence detection system (Applied Biosystems), and relative mRNA expression was calculated by comparative $\mathrm{Ct}$ method.

\section{In vitro proliferation and colony formation assay}

One thousand cells per well were plated and adhered in 96-well plates. Cell growth curves were assessed by Cell Counting Kit-8 (Dojindo) for 5 days. Dissolved agar was used in colony formation assay. Two milliliter of $0.4 \%$ $0.6 \%$ agar was added into 6-well plate as bottom gel. One thousand cells suspended in $4 \mathrm{ml}$ of $0.2 \%$ diluted agar were poured on bottom gel, and were cultured for 2 weeks. MTT was used to staining.

\section{Wound healing and transwell assay}

Cells were plated in 12-well plates with concentration more than $90 \%$. Wounds were scratched by pipette tips and photographed every $24 \mathrm{~h}$. Suspended cells were washed by PBS buffer. Serum-free medium was used for cell culture.

In invasion assay, $100 \mu \mathrm{l}$ of diluted Matrigel (BD Biosciences) was used to coat the chambers' membrane ( $8 \mu \mathrm{m}$ for 24-well plate, Millipore). Fifty thousand cells in $100 \mu \mathrm{l}$ serum-free medium were added into the upper chambers, with full medium in lower chambers, then, cultured for $48 \mathrm{~h}$. For migration assay, cells were cultured for $24 \mathrm{~h}$ under the same conditions without Matrigel. Cells were fixed by $10 \%$ formalin, and stained by $0.1 \%$ crystal violet, and were photographed and counted by inverted microscope.

\section{Immunofluorescence}

Cells were seeded and cultured on glass slides. After $24 \mathrm{~h}$, cells were fixed by $4 \%$ paraformaldehyde for $30 \mathrm{~min}$, penetrated by $0.1 \%$ Triton X-100 for 15 min, and blocked by $5 \%$ BSA for $30 \mathrm{~min}$. Then, cells was incubated with anti-actin antibody (1:80, Acti-stain ${ }^{\mathrm{Tm}} 555$ Fluorecent Phalloidin, Cytoskeleton) for $45 \mathrm{~min}$, and mounted by VECTASHIELD Mounting Medium with DAPI (Vector Labs). The images were obtained by inverted ZEISS LSM710 confocal microscope (40× oil lens) (Carl Zeiss), with ZEN 2009 Light Edition software (Carl Zeiss).

\section{Subcutaneous nude mouse xenografts}

Eight 4-week-old male BALB/c nude mice (Institute of Zoology, China Academy of Sciences) were randomly divided into 2 groups (4 for each group). One million SGC7901/Vector or SGC-7901/shMTA2 cells in $100 \mu \mathrm{l}$ PBS were inoculated subcutaneously. Tumor nodules were measured every 7 days after its length reached over $4 \mathrm{~mm}$, and was calculated by following formula: $V=\left(W_{i d t h}^{2} \times\right.$ Length)/2 [35]. Xenografts were collected at 5th week for immunohistochemistry staining and protein extraction. 


\section{Pulmonary metastases models}

Twenty 4-week-old male BALB/c nude mice (Institute of Zoology, China Academy of Sciences) were randomly divided into 2 groups (10 for each group). One million SGC-7901/Vector or SGC-7901/shMTA2 cells in $100 \mu \mathrm{l}$ PBS were injected by tail vein, respectively. One mouse from each group was sacrificed at one month after injection to evaluate the metastasis formation. Two months later, all mice were sacrificed. Pulmonary metastases were checked by gross specimens and microscopy.

\section{Chromatin immunoprecipitation (ChIP)}

The ChIP assay was performed following the protocol of chromatin immunoprecipitation kit (Millipore). Protein and DNA was crosslinked by formaldehyde, extracted by SDS lysis buffer, and sheared by sonication. Sp1 antibody (Santa Cruz) was used in immunoprecipitation. After purification of precipitated DNA, PCR was performed. The primers used for PCR were listed in Additional file 1: Table S2. PCR products were fractionated by gel electrophoresis and photographed.

\section{Luciferase reporter plasmid, site specific mutagenesis and promoter activity analysis}

Primers for amplification of MTA2 promoter were listed in Additional file 1: Table S3, with Kpn I site at 5' end of forward primer and Bgl II site at reverse primer. MTA2 promoter fragments were amplified from human genomic DNA, and were inserted into pGL3-Basic vector. Six reporter plasmids were constructed and named as pGL31179, pGL3-1042, pGL3-797, pGL3-482, pGL3-117 and pGL3-264. Single-tube luminometer (Promega) was used for dual-luciferase reporter assay (Promega) following the manual.

\section{Statistics}

Chi-square test was used to analyze the categorical variables. Correlation between MTA2 and Sp1 expression was analyzed by Spearman test. Log-rank test in Kaplan-Meier method and Cox regression test was used to assess patients' outcome and prognostic factors. Independent-samples $t$ test was used in quantitive data analysis. $P$-value $<0.05$ was considered as statistical significant. All tests were preformed by SPSS 13.0 software (SPSS Inc.).

\section{Additional file}

Additional file 1: Figure S1. Survival curve of Patients. Patients have no survival difference with different MTA2 status in identical TNM staging. Figure S2. MTA2 expression and migration ability of AGS/shMTA2 cells. MTA2 expression was down-regulated in AGS/shMTA2 cells and was rescued by transient transfection of MTA2 plasmid. Migration ability of AGS/shMTA2 cells was partially recovered. Figure S3. MTA2 expression in xenografts was detected by western blot. MTA2 expression was downregulated in xenografts of SGC-7901/shMTA2 cells. Figure S4. Expression of MTA2 and Sp1 in gastric cancer cell lines. Concomitant expression of MTA2 and Sp1 was observed in gastric cancer cell lines. Figure S5. MTA2 mRNA and protein expressions in Sp1 over-expressed MKN45 cells. MTA2 mRNA expression was increased after Sp1 overexpression in MKN45 cells, whereas the protein level was not significantly changed. Figure S6. p53 expression in MTA2 knockdown cells. p53 expression was increased in SGC-7901/shMTA2 and AGS/shMTA2 cells. Table S1. Clinicopathological characteristics of 127 gastric cancer patients. Table S2. Primers for PCR in chromatin immunoprecipitation. Table S3. Primers for construction of luciferase reporter plasmid.

\section{Abbreviations}

MTA2: Metastasis-associated tumor gene family 2; NuRD: Nucleosome remodeling and deacetylase; Sp1: Specificity protein 1; OS: Overall survival; IHC: Immunohistochemistry; AJCC: American joint committee on cancer; qRTPCR: Quantitative reverse transcription-polymerase chain reaction;

ChIP: Chromatin immunoprecipitation.

\section{Competing interests}

The authors declare that they have no competing interests.

\section{Authors' contributions}

Conceived and designed the experiments: CZ, JZ, BL, YY and ZZ. Performed the experiments: JJ, MS, QC and XC. Analyzed the data: CZ, JJ and JZ. Wrote the manuscript: CZ and JZ. All authors read and approved the final manuscript.

\section{Acknowledgments}

This study was supported by grants from the National Science Foundation of China (81372645, 30801371, 81172329 and 81372644), Shanghai Natural Science Foundation from municipal government (13ZR1425900), the Chinese National High Tech Program (2012AA02A504, 2012AA02A203 and 2011ZX09307-001-05), International Cooperative Project from Shanghai Science and Technology Commission (12410706400).

Received: 15 May 2013 Accepted: 5 September 2013

Published: 8 September 2013

\section{References}

1. Yang G, Wang Y, Zeng Y, Gao GF, Liang X, Zhou M, Wan X, Yu S, Jiang Y, Naghavi M, et al: Rapid health transition in China, 1990-2010: findings from the Global Burden of Disease Study 2010. Lancet 2013, 381:1987-2015.

2. Brenner $H$, Rothenbacher D, Arndt V: Epidemiology of stomach cancer. Methods Mol Biol 2009, 472:467-477.

3. Long N, Moore MA, Chen W, Gao CM, Lai MS, Mizoue T, Oyunchimeg D, Park S, Shin HR, Tajima K, et al: Cancer epidemiology and control in North-East Asia past, present and future. Asian Pac J Cancer Prev 2010, 11(Suppl 2):107-148.

4. Zhao P, Dai M, Chen W, Li N: Cancer trends in China. Jpn J Clin Oncol 2010, 40:281-285

5. Li ZX, Kaminishi M: A comparison of gastric cancer between Japan and China. Gastric Cancer 2009, 12:52-53.

6. Palmer TD, Ashby WJ, Lewis JD, Zijlstra A: Targeting tumor cell motility to prevent metastasis. Adv Drug Deliv Rev 2011, 63:568-581.

7. Valastyan S, Weinberg RA: Tumor metastasis: molecular insights and evolving paradigms. Cell 2011, 147:275-292.

8. Toh Y, Nicolson GL: The role of the MTA family and their encoded proteins in human cancers: molecular functions and clinical implications. Clin Exp Metastasis 2009, 26:215-227.

9. Hofer MD, Menke A, Genze F, Gierschik P, Giehl K: Expression of MTA1 promotes motility and invasiveness of PANC-1 pancreatic carcinoma cells. Br J Cancer 2004, 90:455-462.

10. Qian H, Lu N, Xue L, Liang X, Zhang X, Fu M, Xie Y, Zhan Q, Liu Z, Lin C: Reduced MTA1 expression by RNAi inhibits in vitro invasion and migration of esophageal squamous cell carcinoma cell line. Clin Exp Metastasis 2005, 22:653-662.

11. Zhang $Y, \mathrm{Ng} \mathrm{HH}$, Erdjument-Bromage $H$, Tempst $P$, Bird A, Reinberg D: Analysis of the NuRD subunits reveals a histone deacetylase core complex and a connection with DNA methylation. Genes Dev 1999 13:1924-1935.

12. Manavathi $B$, Singh $K$, Kumar R: MTA family of coregulators in nuclear receptor biology and pathology. Nucl Recept Signal 2007, 5:e010. 
13. Xia L, Zhang Y: Sp1 and ETS family transcription factors regulate the mouse Mta2 gene expression. Gene 2001, 268:77-85.

14. Yao JC, Wang L, Wei D, Gong W, Hassan M, Wu TT, Mansfield P, Ajani J, Xie K: Association between expression of transcription factor Sp1 and increased vascular endothelial growth factor expression, advanced stage, and poor survival in patients with resected gastric cancer. Clin Cancer Res 2004, 10:4109-4117.

15. Zhang J, Zhu ZG, Ji J, Yuan F, Yu YY, Liu BY, Lin YZ: Transcription factor $\mathrm{Sp} 1$ expression in gastric cancer and its relationship to long-term prognosis. World I Gastroenterol 2005, 11:2213-2217.

16. Zhou CF, Ji J, Yuan F, Yu YY, Liu BY, Zhang J, Zhu ZG: [Expression of metastasis associated 1 family member 2 (MTA2) in gastric cancer and its relationship with transcription factor Sp1]. Zhonghua Zhong Liu Za Zhi 2012, 34:592-595.

17. Gallinari P, Di Marco S, Jones P, Pallaoro M, Steinkuhler C: HDACs, histone deacetylation and gene transcription: from molecular biology to cancer therapeutics. Cell Res 2007, 17:195-211.

18. Ji Y, Zhang P, Lu Y, Ma D: Expression of MTA2 gene in ovarian epithelial cancer and its clinical implication. J Huazhong Univ Sci Technolog Med Sci 2006, 26:359-362.

19. Lee H, Ryu SH, Hong SS, Seo DD, Min HJ, Jang MK, Kwon HJ, Yu E, Chung $\mathrm{YH}$, Kim KW: Overexpression of metastasis-associated protein 2 is associated with hepatocellular carcinoma size and differentiation. J Gastroenterol Hepatol 2009, 24:1445-1450.

20. Liu SL, Han Y, Zhang Y, Xie CY, Wang EH, Miao Y, Li HY, Xu HT, Dai SD: Expression of metastasis-associated protein 2 (MTA2) might predict proliferation in non-small cell lung cancer. Target Oncol 2012, 7:135-43.

21. Fu J, Qin L, He T, Qin J, Hong J, Wong J, Liao L, Xu J: The TWIST/Mi2/NuRD protein complex and its essential role in cancer metastasis. Cell Res 2011, 21:275-289.

22. Hall A: The cytoskeleton and cancer. Cancer Metastasis Rev 2009, 28:5-14.

23. Mierke $C T$, Bretz N, Altevogt P: Contractile forces contribute to increased glycosylphosphatidylinositol-anchored receptor CD24-facilitated cancer cell invasion. J Biol Chem 2011, 286:34858-34871.

24. Baumann P, Cremers N, Kroese F, Orend G, Chiquet-Ehrismann R, Uede T, Yagita H, Sleeman JP: CD24 expression causes the acquisition of multiple cellular properties associated with tumor growth and metastasis. Cancer Res 2005, 65:10783-10793.

25. Zhou X, Liu Y, You J, Zhang H, Zhang X, Ye L: Myosin light-chain kinase contributes to the proliferation and migration of breast cancer cells through cross-talk with activated ERK1/2. Cancer Lett 2008, 270:312-327.

26. Thullberg M, Stromblad S: Anchorage-independent cytokinesis as part of oncogenic transformation? Cell Cycle 2008, 7:984-988

27. Reddig PJ, Juliano RL: Clinging to life: cell to matrix adhesion and cell survival. Cancer Metastasis Rev 2005, 24:425-439.

28. Wang LH: Molecular signaling regulating anchorage-independent growth of cancer cells. Mt Sinai J Med 2004, 71:361-367.

29. Luo J, Su F, Chen D, Shiloh A, Gu W: Deacetylation of p53 modulates its effect on cell growth and apoptosis. Nature 2000, 408:377-381.

30. Wierstra I: Sp1: emerging roles-beyond constitutive activation of TATAless housekeeping genes. Biochem Biophys Res Commun 2008, 372:1-13.

31. Li L, Davie JR: The role of Sp1 and Sp3 in normal and cancer cell biology. Ann Anat 2010, 192:275-283.

32. Mansilla S, Portugal J: Sp1 transcription factor as a target for anthracyclines: effects on gene transcription. Biochimie 2008, 90:976-987.

33. Nicolas M, Noe V, Jensen KB, Ciudad CJ: Cloning and characterization of the 5 '-flanking region of the human transcription factor Sp1 gene. J Biol Chem 2001, 276:22126-22132.

34. Lee TI, Young RA: Transcriptional regulation and its misregulation in disease. Cell 2013, 152:1237-1251.

35. Jacob D, Davis J, Fang B: Xenograftic tumor models in mice for cancer research, a technical review. Gene Ther Mol Biol 2004, 8:213-219.

doi:10.1186/1476-4598-12-102

Cite this article as: Zhou et al:: MTA2 promotes gastric cancer cells invasion and is transcriptionally regulated by Sp1. Molecular Cancer 2013 12:102

\section{Submit your next manuscript to BioMed Central and take full advantage of:}

- Convenient online submission

- Thorough peer review

- No space constraints or color figure charges

- Immediate publication on acceptance

- Inclusion in PubMed, CAS, Scopus and Google Scholar

- Research which is freely available for redistribution

Submit your manuscript at www.biomedcentral.com/submit
Biomed Central 